ARTICLE

DOI: 10.1038/s41467-017-01164-5

\title{
Replication fork reversal triggers fork degradation in BRCA2-defective cells
}

Sofija Mijic ${ }^{1}$, Ralph Zellweger ${ }^{1}$, Nagaraja Chappidi ${ }^{1}$, Matteo Berti ${ }^{1}$, Kurt Jacobs ${ }^{1}$, Karun Mutreja ${ }^{1}$, Sebastian Ursich ${ }^{1}$, Arnab Ray Chaudhuri ${ }^{2,3}$, Andre Nussenzweig ${ }^{2}$, Pavel Janscak ${ }^{1} \&$ Massimo Lopes ${ }^{1}$

Besides its role in homologous recombination, the tumor suppressor BRCA2 protects stalled replication forks from nucleolytic degradation. Defective fork stability contributes to chemotherapeutic sensitivity of BRCA2-defective tumors by yet-elusive mechanisms. Using DNA fiber spreading and direct visualization of replication intermediates, we report that reversed replication forks are entry points for fork degradation in BRCA2-defective cells. Besides MRE11 and PTIP, we show that RAD52 promotes stalled fork degradation and chromosomal breakage in BRCA2-defective cells. Inactivation of these factors restores reversed fork frequency and chromosome integrity in BRCA2-defective cells. Conversely, impairing fork reversal prevents fork degradation, but increases chromosomal breakage, uncoupling fork protection, and chromosome stability. We propose that BRCA2 is dispensable for RAD51-mediated fork reversal, but assembles stable RAD51 nucleofilaments on regressed arms, to protect them from degradation. Our data uncover the physiopathological relevance of fork reversal and illuminate a complex interplay of homologous recombination factors in fork remodeling and stability.

\footnotetext{
${ }^{1}$ Institute of Molecular Cancer Research, University of Zurich, 8057 Zurich, Switzerland. ${ }^{2}$ Laboratory of Genome Integrity, National Cancer Institute, National Institutes of Health, Bethesda, Maryland 20892, USA. ${ }^{3}$ Present address: Department of Molecular Genetics, Erasmus University Medical Center, 3000 CA Rotterdam, The Netherlands. Correspondence and requests for materials should be addressed to M.L. (email: lopes@imcr.uzh.ch)
} 
B $R C A 1$ and $B R C A 2$ genes represent paradigmatic examples of tumor suppressors, linking genome instability, and cancer susceptibility ${ }^{1}$. Although several nuclear and cytoplasmic functions have been described for these proteins, mutations predisposing to cancer predominantly affect their common function in homologous recombination (HR). BRCA2 biochemical function in HR has been linked to the replacement of the main single-stranded DNA-binding protein RPA with the central recombination factor RAD51, which channels extended ssDNA regions for strand exchange reactions ${ }^{1,2}$. The HR function of BRCA2 has been mostly studied in response to double-strand breaks (DSBs). As a result, both the cancer predisposition and the effectiveness of certain chemotherapeutic drugs associated with $B R C A 2$ deficiencies have long been linked to the DSB-repair defect ${ }^{3}$. However, recent work has uncovered a second, genetically separable function for BRCA2 in protecting stalled replication forks from extensive nucleolytic degradation ${ }^{4}$. This concept was later extended to several additional HR factors, as well as factors mutated in the cancer predisposition syndrome Fanconi anemia $(\mathrm{FA})^{5}$. While controlled nucleolytic degradation of stalled replication forks likely plays a physiological role to

a

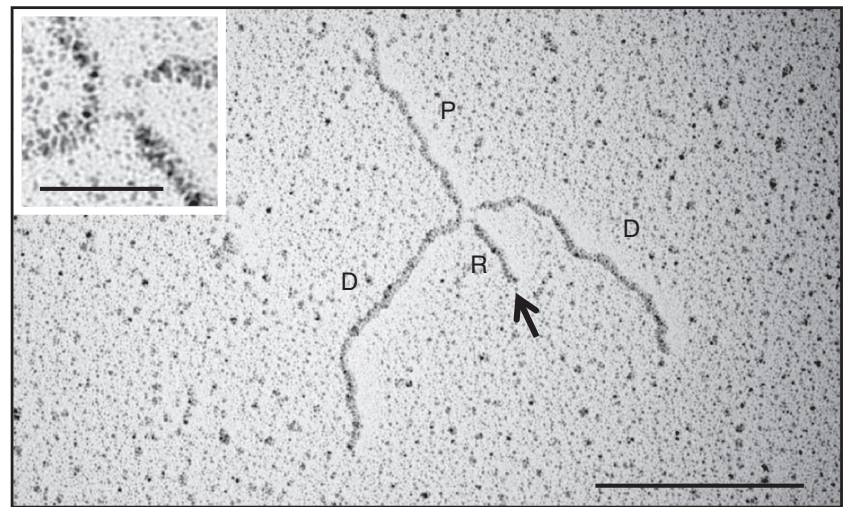

c

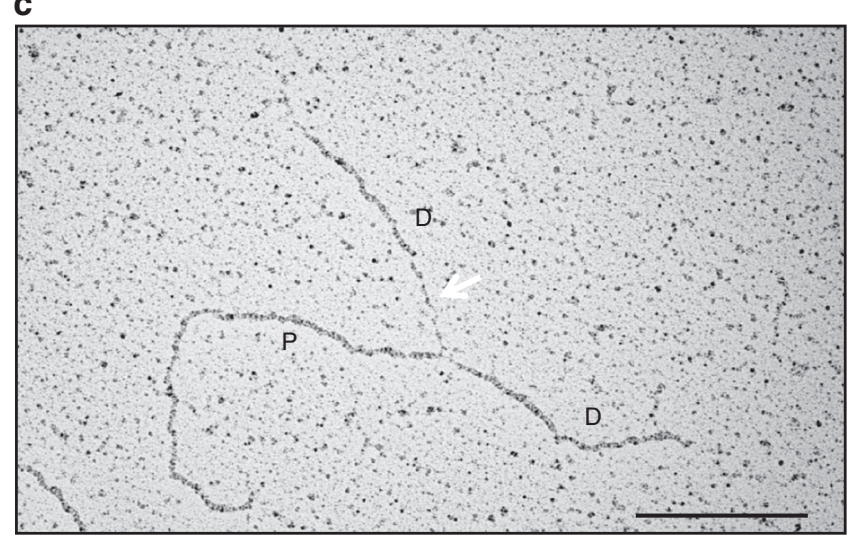

tolerate replication stress, uncontrolled fork degradation upon HR/FA defects is detrimental for genome stability and affects cellular resistance to replication inhibitors ${ }^{4,6-8}$. Most recently, this uncontrolled fork degradation-as opposed to the classical DSB repair defect-was linked both to the lethality of $B R C A 2$ defective embryonic stem cells and to the exquisite sensitivity of BRCA-defective cells to certain chemotherapeutic treatments, elucidating a novel crucial mechanism of therapy resistance of $B R C A$-defective tumors 9 . It is thus of clinical relevance to investigate the detailed molecular mechanisms mediating or limiting fork degradation in response to chemotherapeutic treatments.

Recent visualization of replication intermediates in human cells has revealed replication fork reversal-that is, the conversion of replication forks into four way junctions by strand exchange reactions-as a global, evolutionary conserved cellular response to various conditions of replication stress, such as oncogene activation, chemotherapeutic treatments, and replication of genomic sequences intrinsically prone to form secondary structures ${ }^{10-13}$. Reversed forks were also recently shown to protect genome integrity in unperturbed embryonic stem cells (ESCs), which b

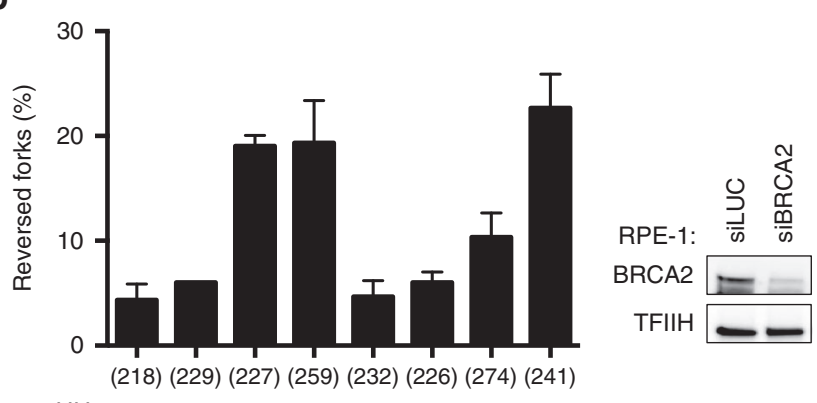

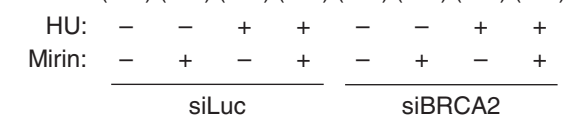

d

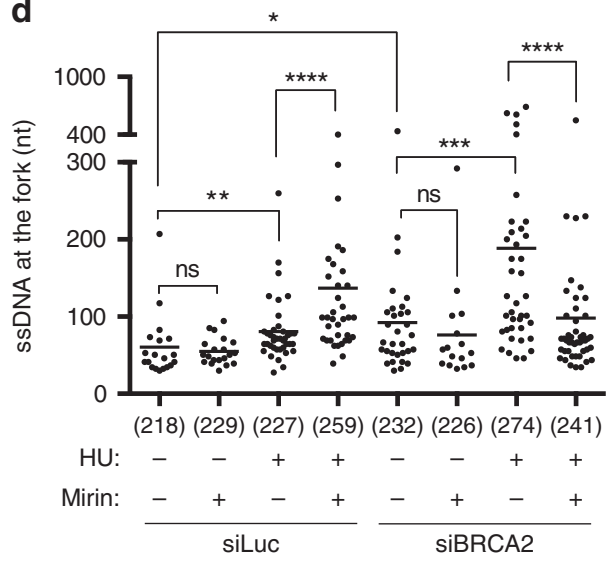

Fig. 1 Stalled replication forks can reverse in the absence of BRCA2, but are targeted by nucleolytic degradation. $\mathbf{a}$, $\mathbf{c}$ Electron micrographs of representative replication forks from RPE-1 cells: parental (P) and daughter (D) duplexes. a The black arrow indicates the regressed arm (R); the four-way junction at the reversed fork is magnified in the inset. c The white arrow points to a ssDNA region at the fork. Scale bar, $200 \mathrm{~nm}(=460 \mathrm{bp}), 40 \mathrm{~nm}(=92 \mathrm{bp})$ in the inset. $\mathbf{b}$ Left panel: frequency of reversed replication forks isolated from mock-depleted (siLuc) and BRCA2-depleted (siBRCA2) RPE-1 cells upon optional $5 \mathrm{~h}$ treatment with $4 \mathrm{mM} \mathrm{HU}$; where indicated, $50 \mu \mathrm{M}$ mirin was added $1 \mathrm{~h}$ before $\mathrm{HU}$ treatment ( $6 \mathrm{~h}$ total treatment). The number of replication intermediates analyzed is indicated in parentheses. The graph depicts mean and SD from three independent EM experiments, blinded to the investigator. The results of the individual biological replicates are in Supplementary Table 1. Right panel: western blot analysis of BRCA2 levels in siLuc and siBRCA2 RPE-1 cells, $48 \mathrm{~h}$ after transfection. TFIIH, loading control. d Graphical distribution of ssDNA length at the junction (white arrow in Fig. 1c) in siLuc and siBRCA2 RPE-1 cells optionally treated with $4 \mathrm{mM}$ of $\mathrm{HU}$ for $5 \mathrm{~h}$ and $50 \mu \mathrm{M}$ of mirin for $6 \mathrm{~h}$. Only molecules with detectable ssDNA stretches are included in the analysis. The lines show the median length of ssDNA regions at the fork in the specific set of analyzed molecules. Statistical analysis: Mann-Whitney test; ns, not significant; ${ }^{\star} P<0.1$; ${ }^{\star \star} P<0.01$; ${ }^{\star \star \star} P<0.001 ;{ }^{\star \star \star \star} P<0.0001$. The number of analyzed molecules is in brackets 
a

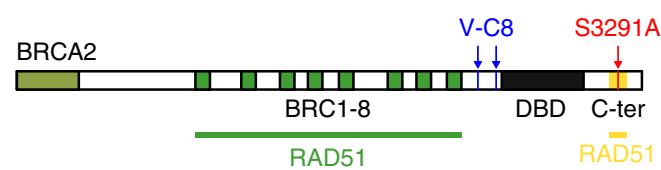

b

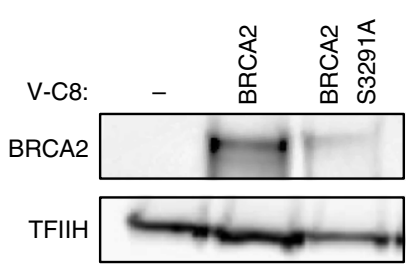

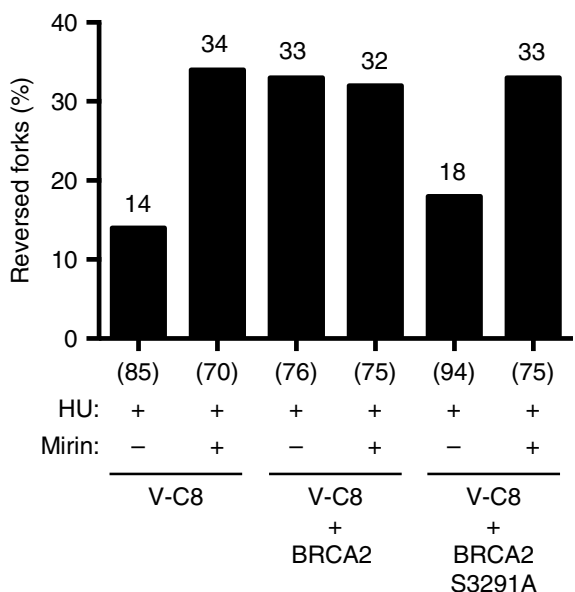

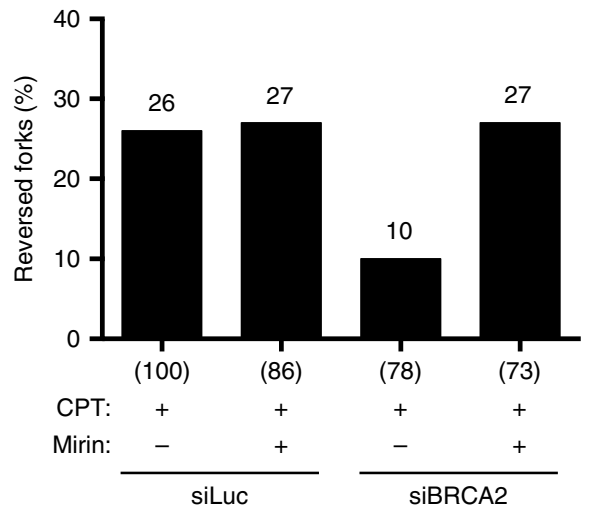

Fig. 2 BRCA2 maintains reversed fork stability in different cell lines and upon different genotoxic treatments. a Top: schematic representation of BRCA2 protein. Green boxes: RAD51-binding BRC repeats; black box: DBD, DNA-binding domain; C-ter, yellow bar: RAD51-biding C-terminal region. Blue arrows indicate truncations in V-C8 cells; the S3291A mutation is marked in red. Bottom: frequency of reversed replication forks isolated from VC-8 cells and V-C8 cells stably expressing full-length BRCA2 or BRCA2 containing the S3291A mutation, treated as in Fig. 1 ( $4 \mathrm{mM} \mathrm{HU}$ for $5 \mathrm{~h}$; $50 \mu \mathrm{M}$ mirin for $6 \mathrm{~h}$ ). The number of analyzed molecules is indicated in brackets. Results of two independent EM experiments are in Supplementary Table 4. Right: western blot analysis of BRCA2 levels in V-C8 and complemented cells. TFIIH, loading control. b EM-based analysis of reversed replication forks isolated from siLuc and siBRCA2 (48 h) RPE-1 cells treated with $25 \mathrm{nM} \mathrm{CPT}$ for $1 \mathrm{~h}$; where indicated, $50 \mu \mathrm{M}$ mirin was added $1 \mathrm{~h}$ before CPT treatment ( $2 \mathrm{~h}$ total treatment). In brackets, the total number of analyzed molecules. Results of two independent EM experiments are in Supplementary Table 5

experience endogenous replication stress as a consequence of their accelerated cell cycle progression ${ }^{14}$. Fork reversal was shown to require the central recombinase $\mathrm{RAD} 51^{11}$, suggesting that classical HR factors mediate strand exchange reactions at replication forks. Furthermore, reversed forks can be restarted by RECQ1-dependent branch migration ${ }^{15}$, but can also undergo controlled resection by DNA2/WRN ${ }^{7}$. The involvement of HR factors in reversed fork formation and processing suggests that fork reversal might be mechanistically linked to the extensive fork degradation observed in BRCA2-defective cells.

Here, we show that replication fork reversal is required for fork degradation in BRCA2-defective cells, as regressed arms act as entry points for MRE11-dependent degradation. Furthermore, we clarify the differential contribution of RAD51 and RAD52 in different steps of fork remodeling and protection. Finally, we provide evidence that, albeit priming fork degradation, reversal of stalled forks is essential to prevent excessive chromosomal breakage in BRCA2-defective tumor cells.

\section{Results}

Unstable reversed forks and extended ssDNA upon BRCA2 defects. To assess replication fork architecture during fork degradation in BRCA2-defective cells, we visualized replication intermediates in vivo by an established electron microscopy (EM) method $^{16}$. We treated untransformed human retinal pigmented epithelial (RPE-1) cells with hydroxyurea (HU) to deplete nucleotides and stall replication forks. As previously shown ${ }^{11}$, the HU treatment led to significant accumulation (20\%) of reversed replication forks, but their frequency was decreased twofold upon BRCA2 depletion by small interfering RNA (siRNA) (Fig. 1a, b; Supplementary Table 1). MRE11 inhibition by $\operatorname{mirin}^{17}$ had no significant effect on reversed fork frequency in untreated cells or in HU-treated wild-type cells, but restored full fork reversal levels in BRCA2-depleted HU-treated cells (Fig. 1b; Supplementary
Table 1). Thus, replication forks can be effectively reversed upon HU treatment also in the absence of BRCA2, but they are targeted by MRE11-dependent degradation. Although we did not detect a specific accumulation of ssDNA on regressed arms in HU-treated BRCA2-defective cells (Supplementary Fig. 1a), extended ssDNA stretches were observed upon $B R C A 2$ downregulation at standard three-way fork junctions and were suppressed by mirin treatment in HU-treated cells (Fig. 1c, d). Shorter HU treatments also led to reduced reversed fork frequency in BRCA2-defective cells, but did not reveal increased ssDNA at fork junctions or regressed arms (Supplementary Fig. 1b, c, Supplementary Table 2). Taken together, these data suggest that nucleolytic processing in HU-treated BRCA2-defective RPE-1 cells rapidly degrades regressed arms and, upon prolonged treatments, continues on newly synthesized DNA behind the fork. Whether or not transient accumulation and/or partial resection of reversed forks is visible by EM upon short genotoxic treatments in BRCA2-defective cells likely reflects different kinetics of fork reversal and processing in different cell lines ${ }^{18}$. These data highlight the different resolution and limitations of DNA fiber assays and EM visualization of fork remodeling and degradation, as recently discussed ${ }^{19}$.

As PTIP depletion in mouse B cells was recently reported to suppress fork degradation in BRCA2-defective cells by limiting MRE11 recruitment at stalled forks ${ }^{9}$, we identified conditions to downregulate PTIP by two different siRNAs in RPE-1 cells, preceding long-term effects on cell cycle progression (Supplementary Fig. 2a, b) ${ }^{20}$, and monitored fork degradation by DNA fibers. As reported ${ }^{4,9}$, HU-treated BRCA2-defective cells displayed marked degradation of nascent DNA, also by a labeling scheme that excludes shortening of replicated tracts by fork breakage (Supplementary Fig. 2c, d). In these conditions, similarly to mirin, PTIP downregulation suppressed fork degradation (Supplementary Fig. 2c) and restored wild-type levels of reversed forks and ssDNA in BRCA2-defective 
HU-treated cells (Supplementary Fig. 2e, f and Supplementary Table 3). Thus, reversed forks appear as "entry points" for extensive MRE11-dependent degradation of stalled forks in BRCA2-defective RPE-1 cells.

Chinese Hamster BRCA2-defective cells (V-C8), previously reported to undergo fork degradation upon $\mathrm{HU}$ treatment ${ }^{4}$, also displayed reduced reversed fork levels and extended ssDNA stretches at forks (Fig. 2a, Supplementary Fig. 3a). Mirin treatment or complementation of these cells with WT BRCA2 restored high frequencies of reversed forks and short ssDNA stretches at forks. However, expression of the BRCA2 phosphorylation mutant S3291A-which causes a defect in fork integrity, but allows HR-mediated DSB repair ${ }^{4}$-failed to complement either defect in V-C8 cells (Fig. 2a, Supplementary Table 4 and Supplementary Fig. 3a), further linking reversed fork instability and fork degradation upon BRCA2 defects. Notably, reduced reversed fork frequency and extended ssDNA stretches at forks-both effectively suppressed by MRE11 inhibition-were also observed in BRCA2-depleted cells upon short treatments with low dose (25 nM) of camptothecin (CPT; Fig. 2b, Supplementary Table 5 and Supplementary Fig. 3b), which induces frequent fork reversal but does not completely arrest fork

\section{a}
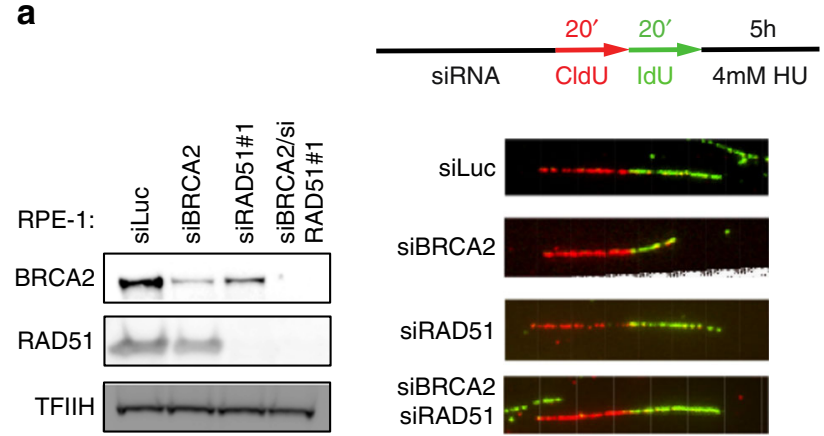

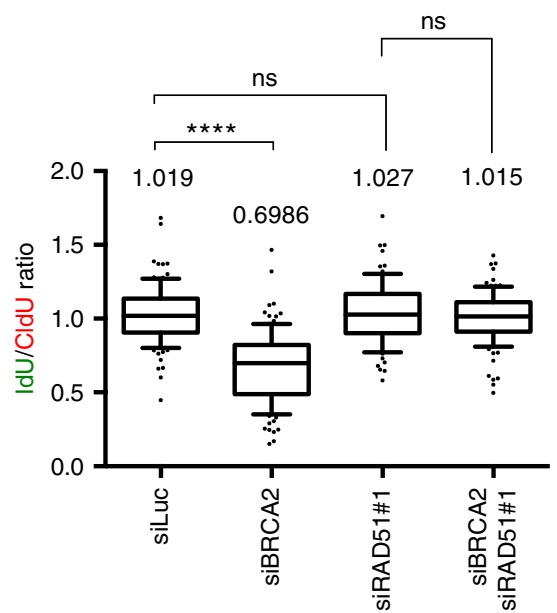

b

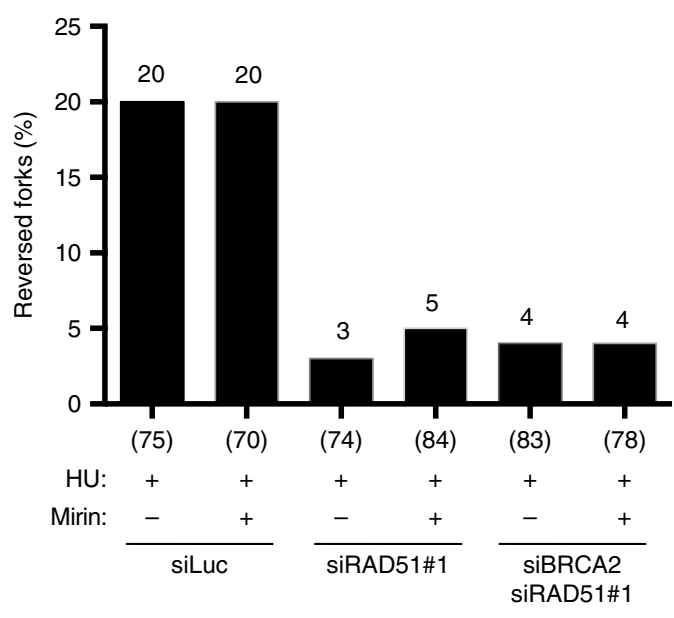

C
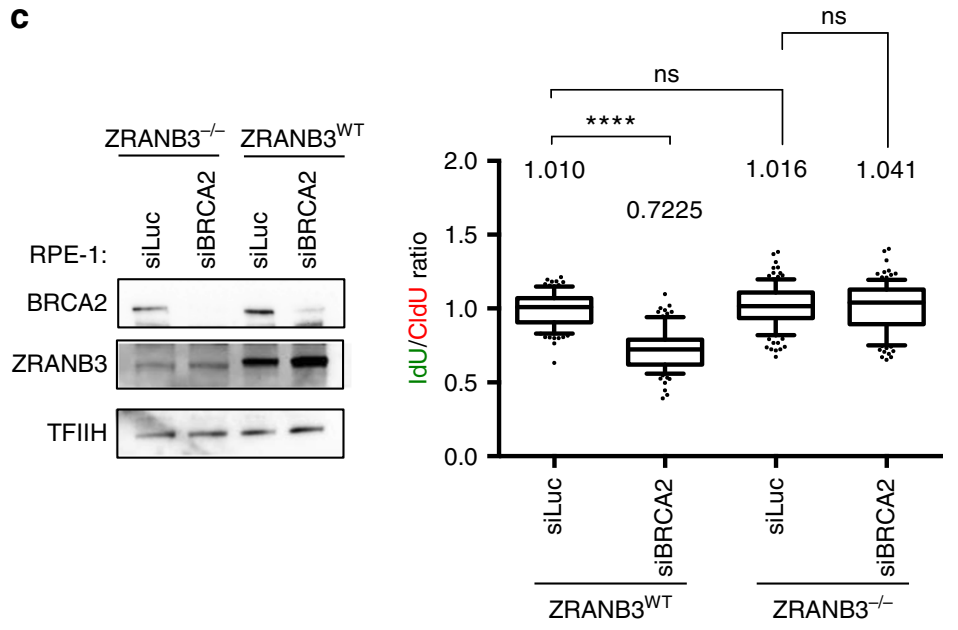

d

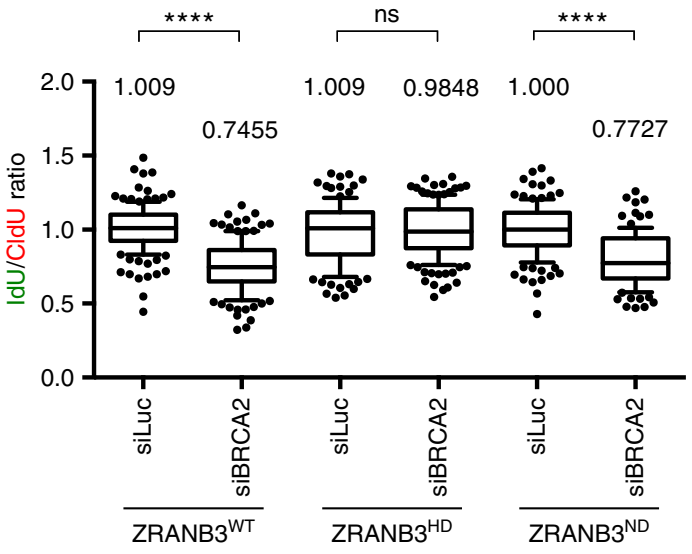

$\mathbf{e}$

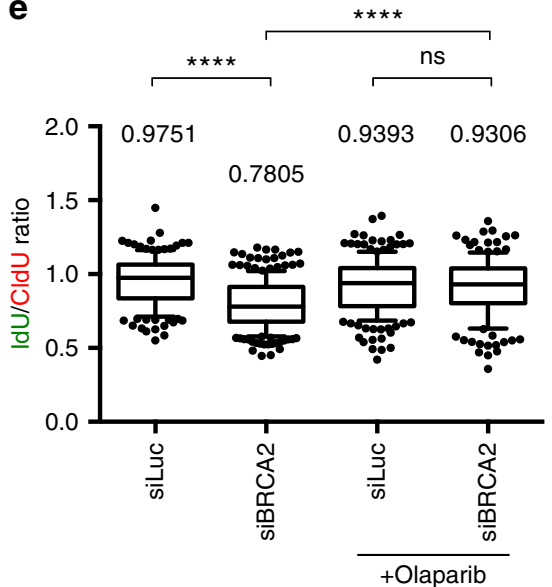


progression $^{11,21}$. These data show that BRCA2 generally protects reversed forks from nucleolytic degradation also in conditions of mild replication interference, where fork degradation is difficult to monitor by DNA fiber assays.

Replication fork reversal is required for fork degradation. The role of BRCA2 in fork protection was previously linked to RAD51 chromatin loading ${ }^{5,22}$. However, RAD51 is also essential for the accumulation of reversed forks, which appear to be the substrate for degradation in BRCA2-defective cells (Figs. 1 and 2). To resolve this conundrum, we analyzed fork degradation upon effective RAD51 downregulation. In contrast to BRCA2 defects ${ }^{4,22}$, depletion of RAD51 by two different siRNA sequences in HU-treated cells did not lead to fork degradation and surprisingly suppressed fork degradation in BRCA2-depleted cells (Fig. 3a, Supplementary Fig. 4a). These data suggest that preventing fork reversal by RAD51 inactivation prevents fork degradation in BRCA2-defective cells. Indeed, in our EM analysis, RAD51 depletion abolished HU-induced fork reversal also in the presence of mirin and prevented mirin-dependent restoration of reversed fork levels in BRCA2-defective cells (Fig. 3b and Supplementary Table 6). Notably, a different genetic perturbation that was recently shown to affect reversed fork formation in vivo ${ }^{23}$-that is, depletion of the DNA translocase ZRANB3 ${ }^{24-26}$-also completely suppressed fork degradation in BRCA2-defective cells (Fig. 3c). Reversed fork formation requires the helicase, but not the nuclease activity of ZRANB3 ${ }^{23}, 24,26$. Accordingly, we found that cells expressing at endogenous levels ${ }^{23}$ helicase-defective-but not wild-type or nuclease-defective-ZRANB3 are resistant to fork degradation upon BRCA2 downregulation (Fig. 3d). Furthermore, PARP inhibition prior to $\mathrm{HU}$ treatment, which was previously reported to prevent efficient fork reversal ${ }^{11}$, also suppressed fork degradation in BRCA2-depleted cells (Fig. 3e). Interestingly, this effect was not reported when the PARP inhibitor was added concomitantly with $\mathrm{HU}^{27}$. The latter conditions are likely to be initially permissive for $\mathrm{HU}$-dependent reversed fork accumulation and thus prime fork degradation before PARP inhibition results in RECQ1-dependent reversed fork resolution ${ }^{11,15}$. Altogether, these results strongly support the notion that fork reversal triggers fork degradation in BRCA2-deficient cells. PARP inhibitor and cisplatin treatments were also recently used to link chemoresistance in BRCA2-defective cells with restored fork stability ${ }^{9,28}$. However, it should be noted that this outcome requires PARP inhibition or downregulation before $B R C A 2$ inactivation ${ }^{9,28}$, under which conditions the efficiency of fork remodeling has not been directly tested.

Fork reversal does not require stable RAD51 nucleofilaments. In light of these data and previous reports ${ }^{4}$, RAD51 seems to be essential both for BRCA2-independent reversed fork formation and for BRCA2-dependent protection of reversed forks from nucleolytic degradation. Thus, different genetic manipulations affecting RAD51 function may have diverse effects on each step of fork remodeling, likely explaining the different fork degradation phenotypes reportedly associated with $R A D 51$ defects $^{4,22}$. A dominant $R A D 51$ mutant allele, found in FA patients, that is, RAD51-T131P, was recently reported to destabilize RAD51 nucleofilaments, by constitutive activation of RAD51 ATPase activity, leading to ssDNA accumulation by nucleolytic processing of replicating $\mathrm{DNA}^{8}$. Upon HU treatment, these patient cells - similarly to BRCA2-deficient cells-displayed extensive fork degradation, which was suppressed by mirin treatment (Fig. 4a). EM analysis of RAD51-T131P cells revealed a marked reduction in reversed fork levels, compared to wild-type counterparts, which was also suppressed by MRE11 inhibition (Fig. $4 \mathrm{~b}$ and Supplementary Table 7). Together with our previous results, these data strongly suggest that unstable RAD51 filaments in RAD51-T131P cells are still capable of driving fork reversal, but fail to protect reversed forks from nucleolytic degradation, uncoupling RAD51 functions in fork remodeling and stability.

RAD52 promotes stalled fork degradation via MRE11 recruitment. As RAD51-mediated fork reversal is BRCA2-independent, we next tested whether RAD52-which was shown to play an essential role in the absence of BRCA2 and to assist HR mechanisms specifically upon replication stress ${ }^{29-31}$ - could assist RAD51 in reversed fork formation and mediate fork degradation upon BRCA2 deficiency. Besides its recently established role in mitotic DNA synthesis and upon breakage of persistently stalled forks ${ }^{31}, 32$, RAD52 is also stably recruited to chromatin in unperturbed $S$ phase ${ }^{32}$ and might thus participate in early events occurring at transiently stalled replication forks. Importantly, RAD52 depletion by two independent siRNA sequences-as well as treatment with a specific RAD52 inhibitor ${ }^{32}, 33$ - completely abolished fork degradation in BRCA2-deficient cells (Fig. 5a), in conditions that do not drastically affect cell cycle progression (Supplementary Fig. 4b). However, differently from RAD51 depletion, RAD52 depletion did not per se affect fork reversal, but rather restored normal reversed fork levels in HU-treated BRCA2-defective cells (Fig. 5b and Supplementary Table 8). These effects are highly reminiscent of those observed for MRE11 inhibition or PTIP depletion (Supplementary Fig. 2) ${ }^{9}$ and suggest a key role for RAD52 in driving MRE11-dependent reversed fork processing. In line with this interpretation, RAD52 inhibition significantly reduced recruitment of MRE11 to HU-stalled forks in BRCA2-defective cells, as monitored by iPOND (Fig. 5c). Furthermore, similarly to MRE11 inhibition and PTIP depletion ${ }^{9}$, RAD52 depletion markedly suppressed HU-induced chromosomal breakage associated with BRCA2 deficiency (Fig. 5d). Thus,

\footnotetext{
Fig. 3 Impairing replication fork reversal prevents fork degradation in BRCA2-defective cells. a RPE-1 cells were transfected with siRNA before CldU (red) and IdU (green) labeling (siBRCA2, $48 \mathrm{~h}$; siRAD51, 24 h), followed by treatment with $4 \mathrm{mM} \mathrm{HU}$ for $5 \mathrm{~h}$. Left panel: levels of indicated proteins, assessed by western blot. TFIIH, loading control. Middle panel: a representative set of DNA fibers from each condition is shown. Right panel: IdU/CldU tract length ratio is plotted. Horizontal lines and the numbers indicate the median value. Whiskers indicate the 10-90 percentiles. At least one hundred replication forks were analyzed for each condition. Statistical analysis: Mann-Whitney test; ns, not significant; ${ }^{\star \star \star \star} P<0.0001$. See also Supplementary Fig. 4a. b Frequency of reversed replication forks isolated from siLuc, siBRCA2 ( $48 \mathrm{~h})$ and siRAD51 (24 h) RPE-1 cells treated as in Fig. 1 ( 4 mM HU for 5 h; $50 \mu M$ mirin for $6 \mathrm{~h}$ ). In brackets, the total number of analyzed molecules. Results of two independent EM experiments are in Supplementary Table 6. c The indicated U2OSbased cell lines were transfected with siRNA before CldU (red) and IdU (green) labeling (siBRCA2, 48 h; siRAD51, 24 h), followed by treatment with 4 mM $\mathrm{HU}$ for $5 \mathrm{~h}$ and DNA fiber spreading. Left panel: levels of indicated proteins, assessed by western blot. TFIIH, loading control. Right panel: IdU/CldU tract length ratio is plotted. Track length analysis and statistics as in Fig. 3a. d Stable derivatives of ZRANB3-KO U2OS cells, expressing wild-type (WT), helicasedead (HD), or nuclease-dead (ND) ZRANB3 at endogenous levels were transfected with siRNA for BRCA2 $48 \mathrm{~h}$ before CldU (red) and IdU (green) labeling, followed by treatment with $4 \mathrm{mM} \mathrm{HU}$ for $5 \mathrm{~h}$. Track length analysis and statistics as in Fig. 3a. e U2OS cells were transfected with the indicated siRNAs $48 \mathrm{~h}$ before $\mathrm{CldU}$ (red) and IdU (green) labeling, followed by treatment with $4 \mathrm{mM} \mathrm{HU}$ for $5 \mathrm{~h}$. The PARP inhibitor olaparib (10 $\mu \mathrm{M}$ ) was optionally added $2 \mathrm{~h}$ before $\mathrm{CldU}$ addition. Track length analysis and statistics as in Fig. 3a
} 
a

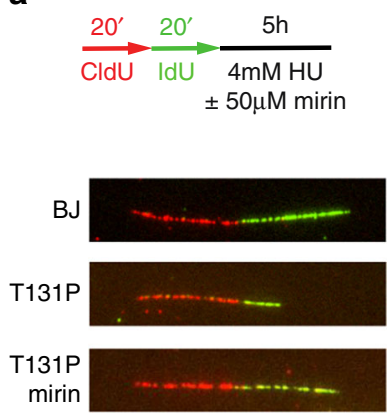

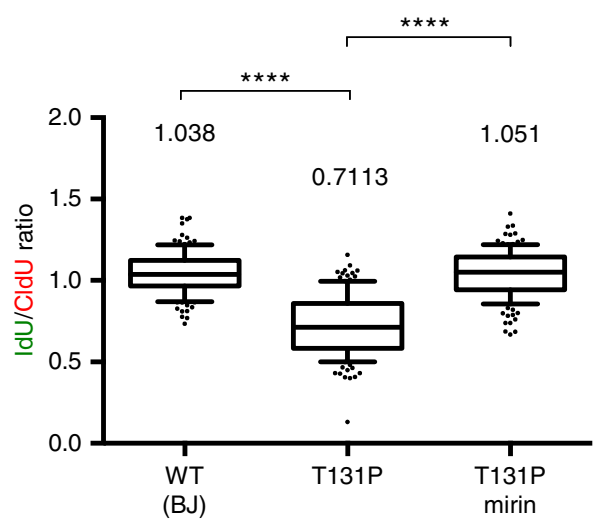

b

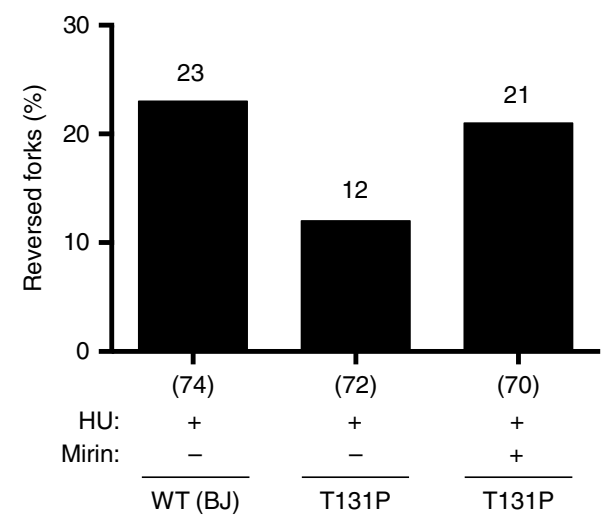

Fig. 4 Stable RAD51 nucleofilaments are required not to form, but rather to protect reversed forks from nucleolytic degradation. a Control (BJ) or RAD51-T131P fibroblasts were labeled with CldU (red) and IdU (green), followed by treatment with $4 \mathrm{mM} \mathrm{HU}$ for $5 \mathrm{~h}$ and $50 \mu \mathrm{M}$ mirin for $6 \mathrm{~h}$, as indicated. A set of representative DNA fibers from each condition is shown. Ratios of IdU vs. CldU tracts are plotted. Track length analysis and statistics as in Fig. $3 a$. b EM-based assessment of the frequency of reversed replication forks isolated from BJ and T131P treated as indicated ( $4 \mathrm{mM} \mathrm{HU}$ for $5 \mathrm{~h}$; $50 \mu \mathrm{M}$ mirin for $6 \mathrm{~h})$. In brackets, the total number of analyzed molecules. Results of two independent EM experiments are in Supplementary Table 7

RAD52 is required to prime MRE11-dependent stalled fork resection in BRCA2-defective cells. Whether the role of RAD52 in MRE11 recruitment and reversed fork resection reflects its strand exchange $^{34}, 35$, single-strand annealing ${ }^{36}$, inverse RNA/DNA strand exchange, ${ }^{37}$ or other yet uncharacterized biochemical activities will require further investigation.

Fork reversal prevents chromosome breakage upon fork stalling. Reversed forks were recently shown to protect against genome instability during accelerated proliferation in early embryogenesis ${ }^{14}$. We thus tested whether the reported rescue of viability of mouse Brca2-null ESCs by PTIP depletion ${ }^{9}$ was also related to the protection of reversed forks from nucleolytic degradation. Upon transient downregulation of $\mathrm{Brca} 2$ in unperturbed mouse ESCs (Supplementary Fig. 4c), we observed a decrease in the level of endogenous reversed forks, as compared to control cells. Notably, rescuing fork degradation by PTIP depletion restored normal frequencies of reversed forks in Brca2-null cells (Fig. 6a and Supplementary Table 9). These data further support a model where the essential role of key HR factors -for example, RAD51 and BRCA2-in early embryogenesis reflects their function in replication fork remodeling and protection 9,14

Preventing MRE11-dependent degradation by mirin treatment, as well as PTIP or RAD52 downregulation, suppressed the chromosomal breakage observed in HU-treated BRCA2-defective cells (Figs. 5d and $6 \mathrm{~b})^{9}$. However, preventing fork reversal-for example, by ZRANB3 inactivation-also suppressed nucleolytic degradation (Fig. 3c), but rather elevated chromosomal breakage in BRCA2-defective U2OS cells. Chromosomal breaks upon simultaneous inactivation of ZRANB3 and BRCA2 were not suppressed by mirin treatment or PTIP depletion, indicating that they are not directly associated with unscheduled fork degradation (Fig. 6b), but likely with defective HR-mediated repair of DSBs arising upon genotoxic stress in the absence of fork reversal. Furthermore, PARP inhibition shortly before HU treatmentwhich is also preventing effective fork reversal ${ }^{11}$-increased chromosomal breakage in BRCA2-defective cells, but did not further increase chromosome instability in ZRANB3-KO BRCA2defective cells, showing epistatic effects of these two means of fork reversal impairment (Fig. 6c). Altogether, these data strongly suggest that preventing fork degradation by abolishing fork reversal is detrimental for genome stability in BRCA2-defective cells, as it likely results in replication fork collapse. Thus, fork reversal limits chromosomal breakage and genome instability at stalled forks, providing additional evidence for the physiological role of this global DNA transaction occurring upon replication stress $^{11,13}$. These data also support a recent alternative model for the specific toxicity of PARP inhibition in BRCA2-defective tumors, where fork reversal suppression by PARP inhibitors ${ }^{11,21}$ underlies the observed increase in fork breakage, requiring BRCA2 classical function in DSB repair ${ }^{13}$.

\section{Discussion}

Taken together, our data reveal a complex interplay of different HR factors in forming and processing reversed forks. We propose that the same apparatus that mediates controlled resection of reversed forks-to allow their effective restart-may become deregulated in BRCA2-defective cells and mediate extensive degradation of reversed forks (Fig. 7). Importantly, we show that these processing events are primed by fork reversal and can potentially occur even upon genotoxic treatments that do not completely block replication fork progression (e.g., mild CPT treatments), which better reflect clinically relevant conditions of replication interference and are anyway strong inducers of fork reversal ${ }^{21}$. RAD51 is clearly involved both in the formation and in the protection of reversed forks. Therefore, the molecular consequences of specific RAD51 mutations will likely depend on residual fork reversal and fork protection activities in each genetic background. Based on our data, stable RAD51 nucleofilaments are strictly required to protect regressed arms, but unstable filaments and/or inefficient loading of RAD51 on ssDNA-as in BRCA2-deficient or RAD51-T131P cells-would not impair strand exchange reactions at replication forks (i.e., fork reversal), probably because they do not imply extensive homology search at a distance. It is intriguing that $B R C A 2$ defects and this specific RAD51 mutation are both associated with FA and it will be crucial to extend this molecular analysis to other FA mutations.

An intriguing implication of our work is the BRCA2independent role of RAD51 in promoting fork reversal. This is in principle surprising, as RPA is known to rapidly bind ssDNA generated at forks and BRCA2 has been clearly implicated in replacing RPA with RAD51 ${ }^{38}$, in order to form a stable nucleofilament. However, previous reports have suggested 
a
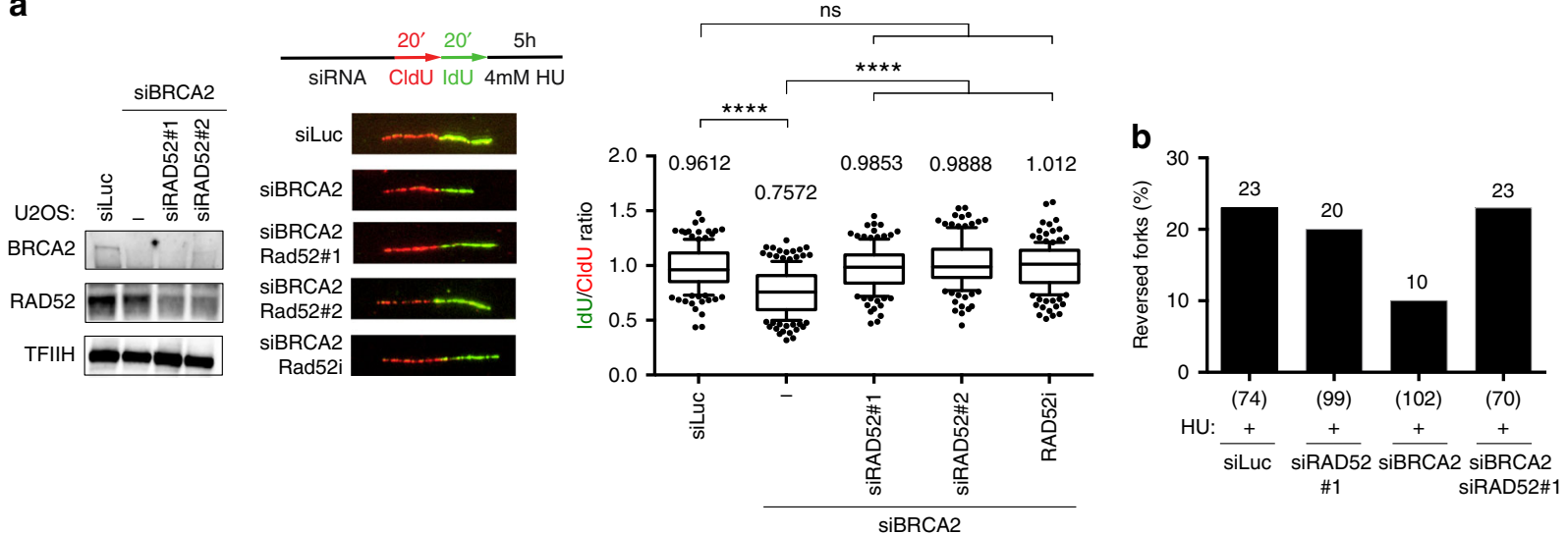

C
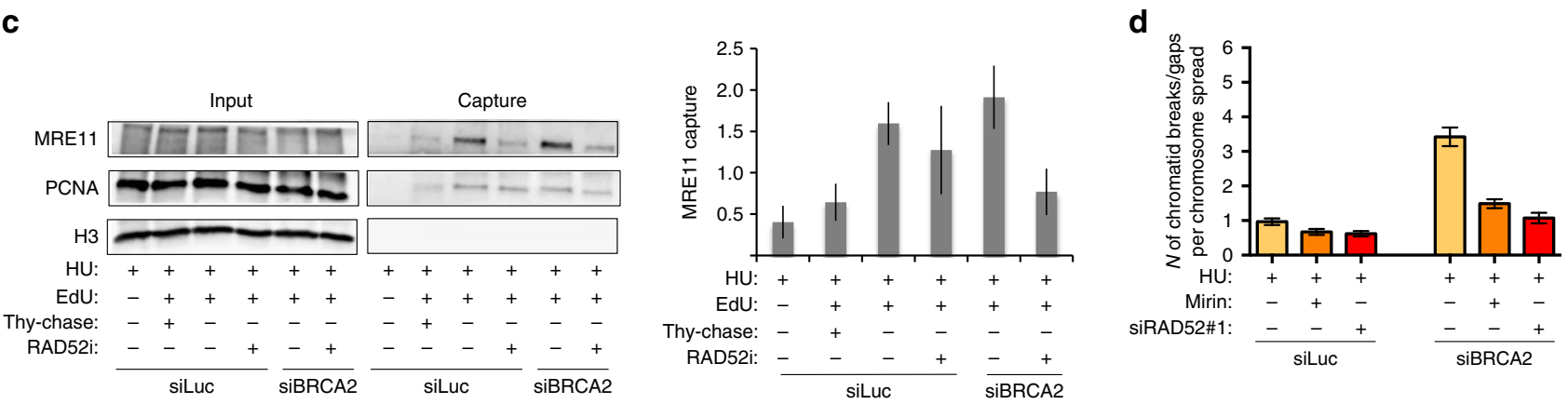

Fig. 5 RAD52 promotes stalled fork degradation in BRCA2-defective cells. a U2OS cells were transfected with siRNA before labeling (siBRCA2, 48 h; siRAD52, $24 \mathrm{~h}$ ) with CldU (red) and IdU (green), followed by treatment with $4 \mathrm{mM} \mathrm{HU}$ for $5 \mathrm{~h}$. The RAD52 inhibitor (AICAR $40 \mu \mathrm{M}$ ) was optionally added $2 \mathrm{~h}$ before CldU labeling. Left: levels of indicated proteins, assessed by western blot. TFIIH, loading control. Middle panel: a representative set of DNA fibers from each condition is shown. Right: ratios of IdU vs. CldU tracts are plotted. Track length analysis and statistics as in Fig. 3a. b EM-based assessment of the frequency of reversed replication forks isolated from U2OS cells transfected with control and siRNA against BRCA2 (48 h) and/or RAD52 (24 h). Cells were treated with $4 \mathrm{mM} \mathrm{HU}$ for $5 \mathrm{~h}$ and $50 \mu \mathrm{M}$ mirin for $6 \mathrm{~h}$, as indicated. In brackets, the total number of analyzed molecules. Results of two independent EM experiments are in Supplementary Table 8. c HEK293T cells were transfected by the indicated siRNAs $48 \mathrm{~h}$ before the EdU-labeling for $15 \mathrm{~min}$ and then treated with HU $4 \mathrm{mM}$ for $5 \mathrm{~h}$. AICAR $40 \mu \mathrm{M}$ (RAD52 inhibitor) was optionally added $2 \mathrm{~h}$ before EdU labeling and retained throughout the experiment. Proteins associated with nascent DNA were isolated by iPOND (see "Methods" section) and detected with the indicated antibodies. For the thymidine chase experiment (Thy-chase), $10 \mu \mathrm{M}$ thymidine was added for $2 \mathrm{~h}$ directly after the EdU labeling. In the control experiment (no EdU), the click reaction is performed using DMSO instead of biotin azide. The graph represents average and SD (error bars) of quantified MRE11 capture signals from three independent experiments. d Chromosomal breakage quantification after $\mathrm{HU}$ and mirin treatment ( $4 \mathrm{mM} \mathrm{HU}$ for $5 \mathrm{~h}$; $50 \mu \mathrm{M}$ mirin for $6 \mathrm{~h}$ ) of $\mathrm{U} 2 \mathrm{OS}$ cells after depletion of BRCA2 $(48 \mathrm{~h})$ and/or RAD52 (24 h). One hundred cells in pro-metaphase were analyzed. Similar results were obtained in two biological replicates

BRCA2-independent RAD51 chromatin loading upon replication stress $^{9,39}$. We envision several non-mutually exclusive scenarios to explain this intriguing observation: (1) besides BRCA2mediated RAD51 loading at DNA ends, alternative mediators may have evolved to assist RPA-RAD51 exchange specifically in the context of ssDNA accumulating at a fork junction; (2) direct displacement of ssDNA-bound RPA by RAD51 at replication forks may be assisted by local exhaustion of free $\mathrm{RPA}^{40}$ and/or reported direct interactions between RAD51 and the replicative helicase; ${ }^{41}$ (3) as suggested by our data on RAD51-T131P mutant cells, inefficient and partial replacement of RPA with short and unstable RAD51 filaments in the absence of BRCA2 may be sufficient to assist strand annealing at uncoupled forks, and thus prime fork reversal, which is anyway assisted by other enzymatic activities $^{23}$. Uncovering the specific regulation of RAD51 activity in fork remodeling will require extensive biochemical reconstitution and in vivo investigations on replication intermediates.

Another important implication of our data is that not all genetic conditions suppressing fork nucleolytic degradation in $B R C A 2$-defective cells are expected to rescue genome stability and survival to genotoxic treatments, which is relevant for informed predictions on chemoresistance of BRCA2-defective tumors.
Based on these data, we would expect that only genetic alterations still allowing reversed fork formation, but preventing their degradation would truly result in resistance to classical chemotherapeutic treatments (Fig. 7). However, due to the involvement of several factors-such as MRE11 and, here, RAD52-in both fork degradation and restart of collapsed forks $^{31,42}$, a detectable decrease in chromosomal breakage due to limited fork resection may not per se predict better recovery and resistance to genotoxic treatments. Indeed, despite extensive resection, BRCA2-defective cells are able to restart stalled forks ${ }^{4,9}$ and a significant proportion of the observed chromosomal breaks may in fact reflect fork restart pathways contributing to cell survival ${ }^{18}$. This intricate series of events likely explains why suppression of fork degradation is observed upon transient RAD52 downregulation in BRCA2-defective cells, although inactivation of these genes is reportedly synthetically lethal ${ }^{29}$ and RAD52 is actively explored as potential therapeutic target in BRCA2-defective tumors ${ }^{43,44}$.

In light of our data and of the structural resemblance of regressed arms to DSBs, it is tempting to speculate that other classical DSB processing and repair factors may play relevant roles in replication fork remodeling, protection, and restart, 
a

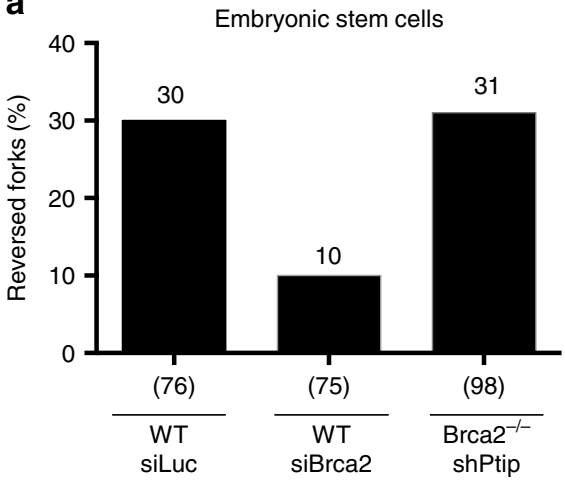

C

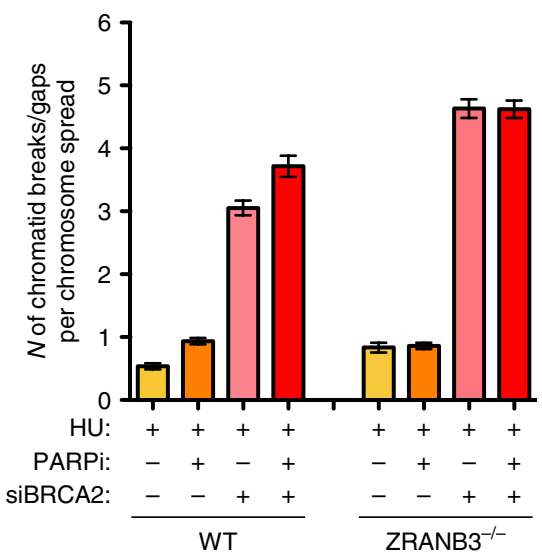

b
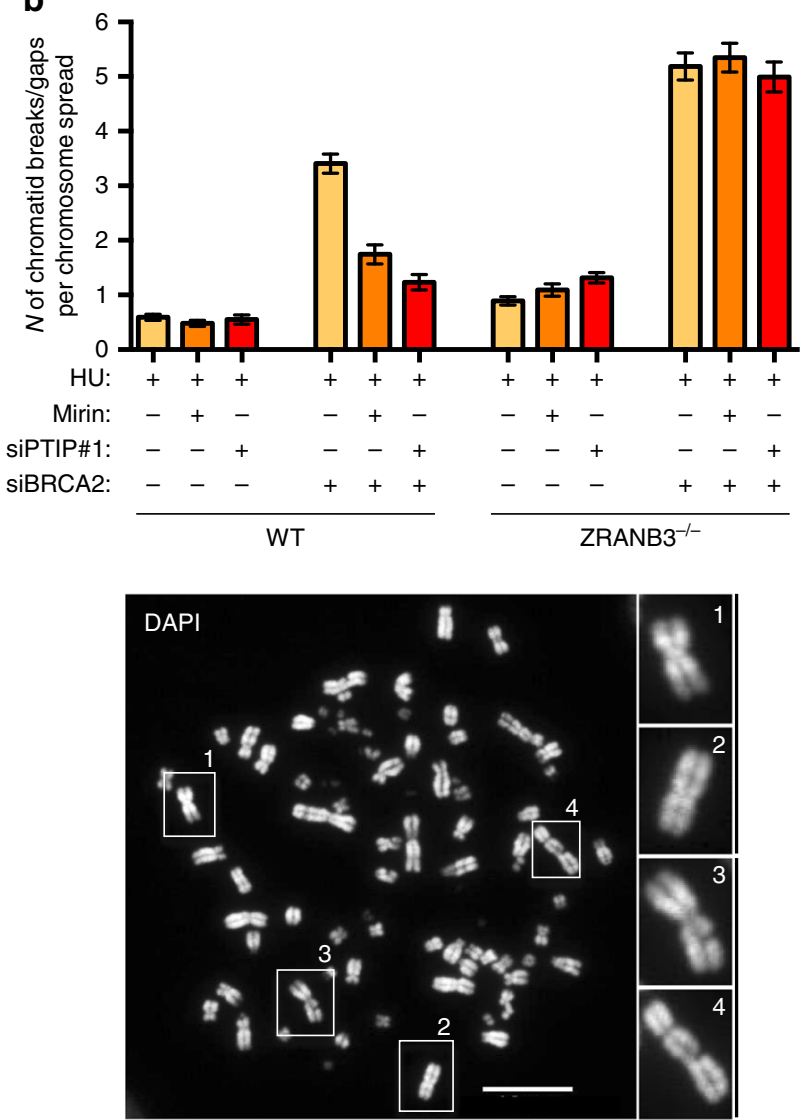

Fig. 6 Fork reversal impairment suppresses fork degradation, but increases chromosomal breakage in BRCA2-defective cells. a Frequency of reversed replication forks isolated from unperturbed mouse ESCs-transfected with siluc or siBrca2 (48 h)-and from Brca2 ${ }^{-/-}$shPtip ESCs. In brackets, the total number of analyzed molecules. Results of two independent EM experiments are in Supplementary Table 9. b Representative count of chromatid breaks upon $5 \mathrm{~h}$ treatment with $\mathrm{HU} 4 \mathrm{mM}$ in control and ZRANB3 knockout (KO) U2OS cells; where indicated, $50 \mu \mathrm{M}$ mirin was added $1 \mathrm{~h}$ before $\mathrm{HU}$ treatment ( $6 \mathrm{~h}$ total treatment), and siRNA transfection was performed $48 \mathrm{~h}$ (BRCA2) or $20 \mathrm{~h}$ (PTIP) before HU treatment. The number of chromatid breaks per chromosome spread was plotted. At least 150 chromosome spreads were analyzed. Error bars represent SEM. A representative DAPI stained chromosome spread is shown. Insets 1 and 2 show intact chromosomes, while 3 and 4 display chromosomal breaks. c Chromosomal breakage quantification of HUtreated ( $4 \mathrm{mM} \mathrm{HU}, 5 \mathrm{~h}$ ) U2OS cells after optional depletion of BRCA2 ( $48 \mathrm{~h}$ ) and/or PARP inhibition (Olaparib $10 \mu \mathrm{M}$, added $2 \mathrm{~h}$ before HU). At least 180 cells in pro-metaphase were analyzed. The number of chromatid breaks per chromosome spread was plotted. Error bars represent SEM. Similar results were obtained in three biological replicates

thereby determining sensitivity or resistance to current chemotherapeutic treatments.

\section{Methods}

Cells and cell culture. Human osteosarcoma U2OS cells, retinal pigment epithelium RPE-1 cells and VC-8 hamster cells, V-C8 cells complemented with human BACs (V-C8 + BRCA2 and V-C8 + BRCA S3291A) ${ }^{4}$ were cultured in DMEM (41966-029, Life Technologies) supplemented with $10 \%$ (v/v) FBS, $100 \mathrm{U} \mathrm{ml}^{-1}$ penicillin, and $100 \mu \mathrm{g} \mathrm{ml}^{-1}$ streptomycin at $37^{\circ} \mathrm{C}$ and $6 \% \mathrm{CO}_{2}$. Patient fibroblasts RAD51-T131P and BJ foreskin fibroblasts (ATCC) were grown in DMEM (41965-039, Life Technologies) supplemented with 15\% (v/v) FBS, $100 \mathrm{U} \mathrm{ml}^{-1}$ penicillin, and $100 \mu \mathrm{g} \mathrm{ml}^{-1}$ streptomycin at $37^{\circ} \mathrm{C}$ and $6 \% \mathrm{CO}_{2}^{8}$. PL2F2 mouse ESCs were maintained in DMEM with $15 \%$ fetal bovine serum, $0.00072 \% \beta$-mercaptoethanol, $100 \mathrm{U} \mathrm{ml}^{-1}$ penicillin, $100 \mu \mathrm{g} \mathrm{ml}^{-1}$ streptomycin, and $0.292 \mathrm{mg} \mathrm{ml}^{-1} \mathrm{~L}$-glutamine at $37^{\circ} \mathrm{C}$ and $5 \% \mathrm{CO}_{2}{ }^{9}$. ESCs were cultured on feeder cells (mouse embryonic fibroblasts, inactivated with $10 \mathrm{mg} \mathrm{ml}^{-1}$ mitomycin C) for two passages, after they were transferred to feeder-free, gelatinized tissue culture dishes $(0.1 \%$ gelatin from porcine skin, Sigma).

Transfections and treatments. For knockdown experiments, cells were transfected $20-48 \mathrm{~h}$ (as indicated below) prior to sample collection with the indicated siRNA using RNAiMax transfection reagent (Life Technologies) according to the manufacturer's instructions:
siLuc (40 nM; 5'-CGUACGCGGAAUACUUCGAUUdTdT-3'); siBRCA2 ( $48 \mathrm{~h}, 40 \mathrm{nM} ; 5^{\prime}$-UUGACUGAGGCUUGCUCAGUUdTdT-3'); siRAD51\#1 (24 h, $40 \mathrm{nM}$; 5'-GACUGCCAGGAUAAAGCUUdTdT-3'); siRAD51\#2 (24 h, $40 \mathrm{nM}$; 5'-GUGCUGCAGCCUAAUGAGAdTdT-3'); siPTIP\#1 (20 h, $40 \mathrm{nM} ; 5^{\prime}$-AAGGAAGAAGAGGAAGAGGAAdTdT-3'); siPTIP\#2 (20 h, $40 \mathrm{nM}$; 5' UGUUUGCAAUUGCGGAUUAUUdTdT-3'); siRAD52\#1 (24 h,10 nM, ON-TARGETplus Human RAD52 (5893),

Dharmacon);

siRAD52\#2 (48 h, $10 \mathrm{nM}$, s11746 (4392420), Ambion).

Mouse ESCs were passaged in feeder-free conditions and plated in $50 \%$ standard culture medium and 50\% Opti-MEM (Thermo Fisher Scientific) containing RNAiMax transfection reagent (Life Technologies) with the mix of following siRNAs at final concentration of $60 \mathrm{nM}$ for $48 \mathrm{~h}$ :

siBrca2\#1 (5'-UGUUAGGAGAUUCAUCUGGdTdT-3');

siBrca2\#2 (5'-GGCCUAGUCUCAAGAACUCdTdT-3');

siBrca2\#3 (5'-GGAAUUGUAAGGUAGGCUCdTdT-3').

BRCA2 conditional knockout cells with shRNAs against Ptip mRNA ESCs were provided by the lab of A.N ${ }^{9}$

The following reagents were used to treat the cells for the indicated time at the indicated final concentrations before collection: $\mathrm{HU}$ (H8627, Sigma-Aldrich) was prepared in double-distilled $\mathrm{H}_{2} \mathrm{O}$ to obtain a $100 \mathrm{mM}\left(7.6 \mathrm{mg} \mathrm{ml}^{-1}\right)$ stock (freshly made); mirin (M9948, Sigma-Aldrich) was dissolved in DMSO to yield a $50 \mathrm{mM}$ stock, and aliquots were stored at $-80^{\circ} \mathrm{C}$; CPT (C9911, Sigma-Aldrich) was dissolved in DMSO to yield a $20 \mathrm{mM}\left(7 \mathrm{mg} \mathrm{ml}^{-1}\right)$ stock (freshly made); Nocodazole (M1404, Sigma-Aldrich) was prepared in DMSO at the final concentration of 


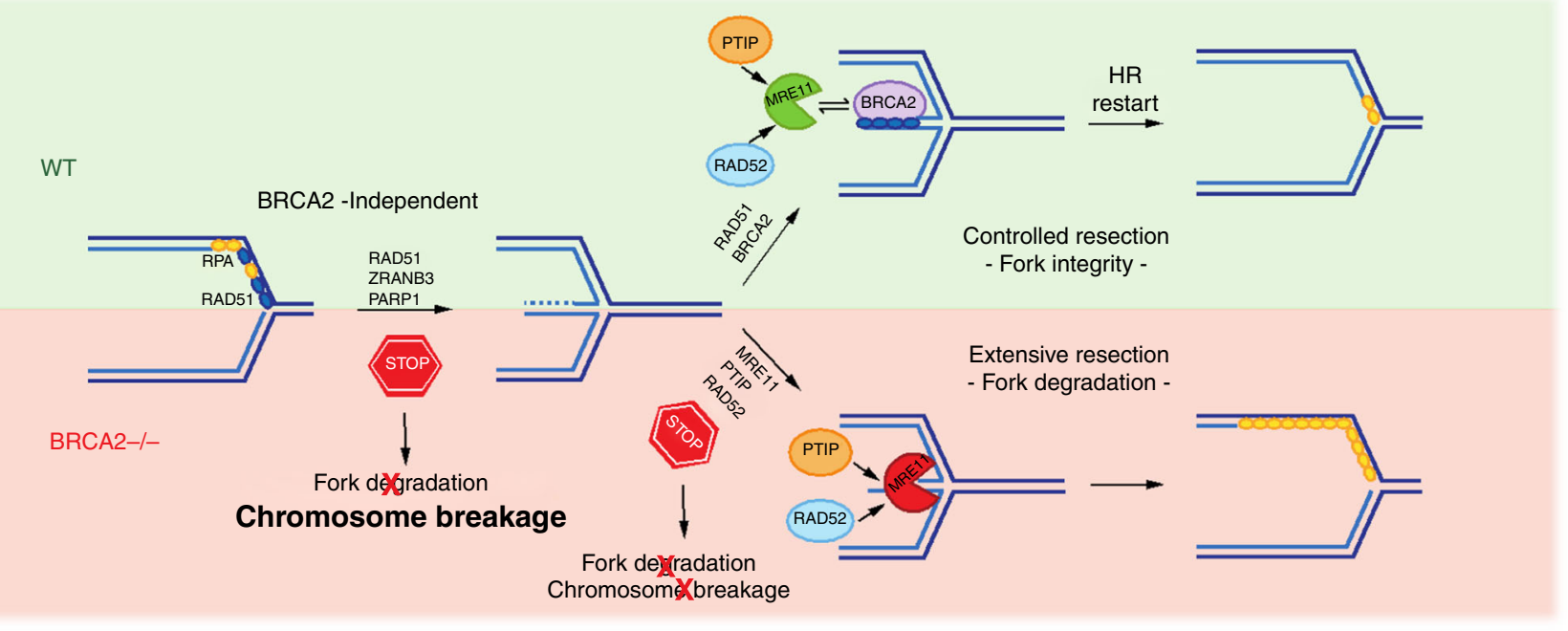

Fig. 7 Model for the role of different HR factors in stalled fork remodeling and protection. With the help of ZRANB3 and PARP activity, RAD51 promotes efficient reversal of stalled replication forks independently of BRCA2. Upon initial resection of reversed forks, RAD51 is efficiently loaded by BRCA2 on regressed arms to limit MRE11/PTIP/RAD52-dependent nucleolytic degradation and promote efficient fork restart. In BRCA2-defective cells, deregulated MRE11-dependent degradation of reversed forks leads to ssDNA accumulation and chromosomal breaks. Limiting reversed fork degradation restores fork integrity and prevents chromosomal breakage. Preventing fork reversal also restores fork integrity in BRCA2-defective cells-by reduced availability of degradation substrates-but leads to increased chromosomal breakage, and is thus detrimental for genome stability

$1 \mathrm{mg} \mathrm{ml}^{-1}$, aliquoted, and stored at $-80^{\circ} \mathrm{C}$. The Rad52 inhibitor (AICAR, A9978, Sigma-Aldrich) was dissolved in $\mathrm{H}_{2} \mathrm{O}$ to a final concentration of $40 \mathrm{mM}$ and stored at $-20^{\circ} \mathrm{C}$. The PARP inhibitor Olaparib (AZD2281, Ku-0059436; S1060 Selleckchem) was prepared in DMSO to obtain the concentration of $20 \mathrm{mM}$, aliquoted, and stored at $-20^{\circ} \mathrm{C}$

Western blotting. Cells were collected using trypsin, immediately lysed using SDS buffer (0.16 M Tris-HCl pH 6.8, $4 \%$ SDS, $20 \%$ glycerol, $100 \mathrm{mM}$ DTT, and $0.01 \%$ bromophenol blue) and sonicated by Bioruptor (Diagenode) at $4{ }^{\circ} \mathrm{C}$ with the highest setting for $10 \mathrm{~min}$ ( $30 \mathrm{~s}$ on and $30 \mathrm{~s}$ off cycles). The lysates were incubated at $70{ }^{\circ} \mathrm{C}$ for $10 \mathrm{~min}$ and centrifuged at 13,000 r.p.m. for 7 min. Protein concentration in samples was measured using Nanodrop (A280). Equal amounts of protein $(50-100 \mu \mathrm{g})$ were loaded on a NuPAGE-Novex 3-8\% Tris-Acetate or NuPAGE-Novex 10\% Bis-Tris gels (Life Technologies) and ran for $1 \mathrm{~h}, 180 \mathrm{~V}$ at room temperature. Proteins were blotted for $100 \mathrm{~min}(30 \mathrm{~V}$, room temperature) on Amersham Protran $0.2 \mathrm{~mm}$ NC (GE Healthcare). Membranes were blocked in $5 \%$ milk in $0.1 \%$ TBST $(1 \times$ TBS supplemented with $0.1 \%$ Tween 20$)$ for at least $30 \mathrm{~min}$ and incubated in $2 \%$ BSA with primary antibodies overnight at $4{ }^{\circ} \mathrm{C}$. Membranes were probed for BRCA2 (1:500, Ab-1, OP 95, EMD Millipore); RAD51 (1:1000, H-92, sc-8349, Santa Cruz Biotechnology); RAD52 (1:1000, F-7, sc-365341, Santa Cruz Biotechnology); PTIP (1:500, ab214732, Abcam); ZRANB3 (1:1000, 23111-1AP, ProteinTech); TFIIH (1:2000, S-19, sc-293, Santa Cruz Biotechnology). Secondary antibodies were added for $1 \mathrm{~h}$ at room temperature (in blocking solution). Membranes were washed three times with $0.1 \%$ TBST, 10 min each, after primary and secondary antibody incubations and detected with ECL detection reagent (GE healthcare). Uncropped blots for each western blot figure are provided in Supplementary Fig. 5.

Electron microscopy analysis. The procedure was performed as recently described $^{16}$, with minor modifications described below. Following the depletion of the protein of interest, asynchronous subconfluent cells were treated with $25 \mathrm{nM}$ CPT for $1 \mathrm{~h}$ or $4 \mathrm{mM} \mathrm{HU}$ for $5 \mathrm{~h}$. Where indicated, cells were pretreated with $50 \mu \mathrm{M}$ mirin for $1 \mathrm{~h}$. Cells were collected, resuspended in PBS, and crosslinked with 4,5', 8-trimethylpsoralen $\left(10 \mu \mathrm{g} \mathrm{ml}^{-1}\right.$ final concentration), followed by irradiation pulses with UV $365 \mathrm{~nm}$ monochromatic light (UV Stratalinker 1800; Agilent Technologies). For DNA extraction, cells were lysed (1.28 M sucrose, $40 \mathrm{mM}$ Tris- $\mathrm{HCl}$ ( $\mathrm{pH} 7.5$ ), $20 \mathrm{mM} \mathrm{MgCl}$, and $4 \%$ Triton X-100; Qiagen) and digested (800 mM guanidine- $\mathrm{HCl}, 30 \mathrm{mM}$ Tris- $\mathrm{HCl}(\mathrm{pH} 8.0), 30 \mathrm{mM}$ EDTA (pH 8.0), $5 \%$ Tween-20, and $0.5 \%$ Triton X-100) at $50^{\circ} \mathrm{C}$ for $2 \mathrm{~h}$ in presence of $1 \mathrm{mg} \mathrm{ml}^{-1}$ proteinase K. The DNA was purified using chloroform/isoamylalcohol (24:1) and precipitated in 0.7 volume of isopropanol. Finally, the DNA was washed with $70 \%$ EtOH and resuspended in $200 \mu \mathrm{l} \mathrm{TE} \mathrm{(Tris-EDTA)} \mathrm{buffer.} \mathrm{Restriction} \mathrm{enzyme} \mathrm{of}$ $100 \mathrm{U}$ (PvuII high fidelity, New England Biolabs) were used to digest $12 \mu \mathrm{g}$ of mammalian genomic DNA for $4-5 \mathrm{~h}$. Replication intermediates enrichment was performed by QIAGEN Plasmid Mini Kit columns. The QIAGEN-tip 20 surface tension was reduced by applying $1 \mathrm{ml}$ QBT buffer. The columns were washed and equilibrated with $10 \mathrm{mM}$ Tris- $\mathrm{HCl}(\mathrm{pH} 8.0), 1 \mathrm{M} \mathrm{NaCl}$, followed by $10 \mathrm{mM}$ Tris- $\mathrm{HCl}(\mathrm{pH} 8.0), 300 \mathrm{mM} \mathrm{NaCl}$, respectively. DNA was then loaded onto the columns. The columns were then washed with high $\mathrm{NaCl}$ solution $(10 \mathrm{mM}$ Tris- $\mathrm{HCl}(\mathrm{pH} 8.0)$ and $900 \mathrm{mM} \mathrm{NaCl})$ and eluted in caffeine solution $(10 \mathrm{mM}$ Tris- $\mathrm{HCl}(\mathrm{pH} 8.0), 1 \mathrm{M} \mathrm{NaCl}$, and $1.8 \%(\mathrm{w} / \mathrm{v})$ caffeine). To purify and concentrate the DNA, an Amicon size-exclusion column was used. DNA was then resuspended in TE buffer. The benzyldimethylalkylammonium chloride method was used to spread the DNA on the water surface and then load it on carbon-coated 400-mesh copper grids. Subsequently, DNA was coated with platinum using a high vacuum evaporator MED 020 (BalTec). Microscopy was performed with a transmission electron microscope (Tecnai G2 Spirit; FEI; LaB6 filament; high tension $\leq 120 \mathrm{kV}$ ) and picture acquisition with a side mount charge-coupled device camera $(2600 \times 4000$ pixels; Orius 1000 ; Gatan, Inc.). For each experimental condition, at least 70 replication fork molecules were analyzed. DigitalMicrograph version 1.83.842 (Gatan, Inc.) and ImageJ (National Institutes of Health) were used to process and analyze the images.

DNA fiber analysis. Following the depletion of proteins of interest, cells were sequentially pulse-labeled with $30 \mu \mathrm{M}$ CldU (c6891, Sigma-Aldrich) and $250 \mu \mathrm{M}$ IdU (I0050000, European Pharmacopoeia) for 20 min and treated with $\mathrm{HU}(4 \mathrm{mM})$ for $5 \mathrm{~h}$. The cells were collected and resuspended in PBS at $2.5 \times 10^{5}$ cells per ml. The labeled cells were diluted 1:5 (v/v) with unlabeled cells, and $2.5 \mu \mathrm{l}$ of cells were mixed with $7.5 \mu$ of lysis buffer ( $200 \mathrm{mM}$ Tris- $\mathrm{HCl}, \mathrm{pH} 7.5,50 \mathrm{mM}$ EDTA, and $0.5 \%(\mathrm{w} / \mathrm{v}) \mathrm{SDS})$ on a glass slide. After $9 \mathrm{~min}$, the slides were tilted at $15-45^{\circ}$, and the resulting DNA spreads were air dried, fixed in 3:1 methanol/acetic acid overnight at $4{ }^{\circ} \mathrm{C}$. The fibers were denatured with $2.5 \mathrm{M} \mathrm{HCl}$ for $1 \mathrm{~h}$, washed with PBS and blocked with $0.2 \%$ Tween 20 in $1 \%$ BSA/PBS for $40 \mathrm{~min}$. The newly replicated CldU and IdU tracks were labeled (for $2.5 \mathrm{~h}$ in the dark, at RT) with anti-BrdU antibodies recognizing CldU (1:500, ab6326; Abcam) and IdU (1:100, B44, 347580; BD), followed by $1 \mathrm{~h}$ incubation with secondary antibodies at RT in the dark: anti-mouse Alexa Fluor 488 (1:300, A11001, Invitrogen) and anti-rat Cy3 (1:150, 712-166-153, Jackson ImmunoResearch Laboratories, Inc.). Fibers were visualized (IX81; Olympus; objective lenses: LC Plan Fluor $60 \times, 1.42$ NA oil Olympus BX60 microscope) and analyzed using ImageJ software. The Mann-Whitney test was applied for statistical analysis using Prism (GraphPad Software).

Isolation of proteins on nascent DNA or iPOND. iPOND was performed essentially as described ${ }^{45}$. At least $1.0 \times 10^{8}$ of HEK293T cells were used per sample. BRCA2 depletion was performed 2 days before EdU labeling. The RAD52 inhibitor (AICAR, $40 \mu \mathrm{M}$ ) was optionally added $2 \mathrm{~h}$ before $10 \mu \mathrm{M}$ EdU labeling ( $15 \mathrm{~min}$ ), followed by $5 \mathrm{~h} 4 \mathrm{mM}$ HU treatment (HU) or by $2 \mathrm{~h} 10 \mu \mathrm{M}$ thymidine chase (Thy-chase). Cells were cross-linked with $1 \%$ formaldehyde for $12 \mathrm{~min}$ at room temperature, quenched with $0.125 \mathrm{M}$ glycine and collected by scraping. The cells were washed with PBS three times and permeabilized with $0.25 \%$ Triton $\mathrm{X}-100 / \mathrm{PBS}$ at room temperature for $30 \mathrm{~min}$. Before the click reaction, samples were washed once in $0.5 \%$ BSA/PBS and once in PBS.

For the conjugation of EdU with biotin azide (Vanderbilt University, Chemical Synthesis Core), cells were incubated with click reaction buffer $(10 \mathrm{mM}$ 
sodium-L-ascorbate, $10 \mu \mathrm{M}$ biotin azide, and $2 \mathrm{mM} \mathrm{CuSO} 4)$ for $2 \mathrm{~h}$ at room temperature. Following the click reaction, cells were washed once in $0.5 \%$ BSA/PBS and once in PBS. Cells were then resuspended in lysis buffer $(50 \mathrm{mM}$ Tris- $\mathrm{HCl}$, $\mathrm{pH} 8.0$, and $1 \%$ SDS) supplemented with protease inhibitors (Roche), and chromatin was solubilized by sonication in a Bioruptor (Diagenode) at $4{ }^{\circ} \mathrm{C}$ for $20 \mathrm{~min}$ ( $20 \mathrm{~s}$ pulse/ $40 \mathrm{~s}$ pause). After centrifugation at $16,100 \times \mathrm{g}$ for $10 \mathrm{~min}$, clarified supernatants were collected and diluted 1:1 (v/v) with PBS containing proteinase inhibitor. To capture biotin-tagged nascent DNA, each sample was incubated at $4{ }^{\circ} \mathrm{C}$ o/n in the dark with streptavidin-agarose beads (Novagen, D00148073). Bead slurry of $200 \mu \mathrm{l}$ was used per $1 \times 10^{8}$ cells. After binding, beads were washed with lysis buffer, followed by one time wash with $1 \mathrm{M} \mathrm{NaCl}$ and two times with lysis buffer. Captured proteins were eluted by boiling beads in $2 \times$ SDS Laemmli Sample Buffer (0.4 g SDS, $2 \mathrm{ml} \mathrm{100 \%} \mathrm{glycerol,} 1.25 \mathrm{ml} 1 \mathrm{M}$ Tris $\mathrm{pH} 6.8$, and $0.01 \mathrm{~g}$ bromophenol blue in $8 \mathrm{ml} \mathrm{H}_{2} \mathrm{O}$ ) for $25 \mathrm{~min}$ at $95^{\circ} \mathrm{C}$. Proteins were resolved by electrophoresis using Mini-PROTEAN TGXTM gels (Bio-Rad) and detected by western blotting with the indicated antibodies: MRE11 (1:2000, NB100-142, Novusbio); PCNA (1:1000, PC10, sc-56), and H3 (1:2000, Ab1791, Abcam).

Analysis of chromosome spreads. After the transfection with specific siRNAs, cells were treated with $4 \mathrm{mM}$ HU for $5 \mathrm{~h}$. The genotoxic agent was removed by washing three times with $1 \times \mathrm{PBS}$ and the cells were then released into fresh medium containing $200 \mathrm{ng} \mathrm{ml}^{-1}$ nocodazole for $16 \mathrm{~h}$. Cells were collected and swollen with $75 \mathrm{mM} \mathrm{KCl}$ for $20 \mathrm{~min}$ at $37^{\circ} \mathrm{C}$. Swollen mitotic cells were collected and fixed with methanol:acetic acid (3:1). The fixing step was repeated two times. Cells were then dropped onto pre-hydrated glass slides and air-dried overnight. The following day, slides were mounted with Vectashield medium containing DAPI. Images were acquired with a microscope (model DMRB; Leica) equipped with a camera (model DFC360 FX; Leica) and visible chromatid breaks/gaps were counted.

Flow cytometry. For flow cytometric analysis of EdU/DAPI, cells were labeled for 30 min with $10 \mu \mathrm{M}$ EdU, collected, and fixed for 15 min with $4 \%$ formaldehyde/ PBS. Cells were washed with $1 \%$ BSA/PBS, pH 7.4 and permeabilized with $0.5 \%$ saponin/1\% PBS. Incorporated EdU was labeled according to the manufacturer's instructions (\#C-10425; Life Technologies). DNA was stained with $1 \mu \mathrm{g} \mathrm{ml}^{-1}$ DAPI. Samples were measured on a Cyan ADP and ATTUNE NXT flow cytometer (Beckman Coulter) and analyzed by the FlowJo software.

Quantitative real-time PCR. Total RNA was isolated from cells using the Oligotex mRNA Mini Kit (Qiagen). RNA of $500 \mathrm{ng}$ was used for complementary DNA (cDNA) synthesis using Transcriptor First Strand cDNA Synthesis Kit (Roche). Quantitative real-time SYBR-Green-based PCR reactions were performed in triplicate and monitored with the Light Cycler 480 (Roche) system. The following primer pairs were used to determine BRCA2 mRNA levels: forward $5^{\prime}$-CACCTCT GGAGCGGACTTATT-3'; reverse 5'-GCTTTGTTGCAGCGTGTCTT-3'.

The housekeeping gene GAPDH, used as a control, was amplified with the following primers: forward 5'-GACATTGTTGCCATCAACGACC-3'; reverse 5'-CCCGTTGATGACCAGCTTCC-3'.

Data availability. The authors declare that all relevant data supporting the findings of this study are available within the article and its supplementary information files or from the corresponding author upon reasonable request.

Received: 11 August 2017 Accepted: 22 August 2017

Published online: 16 October 2017

\section{References}

1. Roy, R., Chun, J. \& Powell, S. N. BRCA1 and BRCA2: different roles in a common pathway of genome protection. Nat. Rev. Cancer 12, 68-78 (2012).

2. Thorslund, $\mathrm{T}$. et al. The breast cancer tumor suppressor BRCA2 promotes the specific targeting of RAD51 to single-stranded DNA. Nat. Struct. Mol. Biol. 17, 1263-1265 (2010).

3. Lord, C. J. \& Ashworth, A. Mechanisms of resistance to therapies targeting BRCA-mutant cancers. Nat. Med. 19, 1381-1388 (2013).

4. Schlacher, K. et al. Double-strand break repair-independent role for BRCA2 in blocking stalled replication fork degradation by MRE11. Cell 145, 529-542 (2011).

5. Schlacher, K., $\mathrm{Wu}, \mathrm{H}$. \& Jasin, $\mathrm{M}$. A distinct replication fork protection pathway connects Fanconi anemia tumor suppressors to RAD51-BRCA1/2. Cancer Cell 22, 106-116 (2012).

6. Berti, M. \& Vindigni, A. Replication stress: getting back on track. Nat. Struct. Mol. Biol. 23, 103-109 (2016).

7. Thangavel, S. et al. DNA2 drives processing and restart of reversed replication forks in human cells. J. Cell Biol. 208, 545-562 (2015)
8. Wang, A. T. et al. A dominant mutation in human RAD51 reveals its function in DNA interstrand crosslink repair independent of homologous recombination. Mol. Cell 59, 478-490 (2015).

9. Ray Chaudhuri, A. et al. Replication fork protection confers chemoresistance in BRCA-deficient cells. Nature 535, 382-387 (2016).

10. Neelsen, K. J., Zanini, I. M. Y., Herrador, R. \& Lopes, M. Oncogenes induce genotoxic stress by mitotic processing of unusual replication intermediates. J. Cell Biol. 200, 699-708 (2013).

11. Zellweger, R. et al. Rad51-mediated replication fork reversal is a global response to genotoxic treatments in human cells. J. Cell Biol. 208, 563-579 (2015).

12. Follonier, C., Oehler, J., Herrador, R. \& Lopes, M. Friedreich's ataxia-associated GAA repeats induce replication-fork reversal and unusual molecular junctions. Nat. Struct. Mol. Biol. doi:https://doi.org/10.1038/nsmb.2520 (2013).

13. Neelsen, K. J. \& Lopes, M. Replication fork reversal in eukaryotes: from dead end to dynamic response. Nat. Rev. Mol. Cell Biol. 16, 207-220 (2015).

14. Ahuja, A. K. et al. A short G1 phase imposes constitutive replication stress and fork remodelling in mouse embryonic stem cells. Nat. Commun. 7, 10660 (2016).

15. Berti, M. et al. Human RECQ1 promotes restart of replication forks reversed by DNA topoisomerase I inhibition. Nat. Struct. Mol. Biol. 20, 347-354 (2013).

16. Zellweger, R. \& Lopes, M. in Genome Instability: Methods and Protocols (eds Muzi-Falconi, M. \& Brown, G.) Methods in Molecular Biology, v.16702. Doi: 10.1007/978-1-4939-7306-4 (Springer Science+Business Media LLC, in the press).

17. Dupré, A. et al. A forward chemical genetic screen reveals an inhibitor of the Mre11-Rad50-Nbs1 complex. Nat. Chem. Biol. 4, 119-125 (2008).

18. Lemacon, D. et al. MRE11 and EXO1 nucleases degrade reversed forks and lead to MUS81-dependent fork rescue in BRCA2-deficient cells. Nat. Commun. 8, doi:10.1038/s41467-017-01180-5 (2017).

19. Vindigni, A. \& Lopes, M. Combining electron microscopy with single molecule DNA fiber approaches to study DNA replication dynamics. Biophys. Chem. 225, 3-9 (2017).

20. Wang, X., Takenaka, K. \& Takeda, S. PTIP promotes DNA double-strand break repair through homologous recombination. Genes Cells 15, 243-254 (2010).

21. Ray Chaudhuri, A. et al. Topoisomerase I poisoning results in PARP-mediated replication fork reversal. Nat. Struct. Mol. Biol. 19, 417-423 (2012).

22. Leuzzi, G., Marabitti, V., Pichierri, P. \& Franchitto, A. WRNIP1 protects stalled forks from degradation and promotes fork restart after replication stress. EMBO J. 35, 1437-1451 (2016).

23. Vujanovic, M. et al. Replication fork slowing and reversal upon genotoxic stress require PCNA polyubiquitination and ZRANB3 DNA translocase activity. Mol. Cell, 67, 882-890 (2017).

24. Ciccia, A. et al. Polyubiquitinated PCNA recruits the ZRANB3 translocase to maintain genomic integrity after replication stress. Mol. Cell 47, 396-409 (2012).

25. Yuan, J., Ghosal, G. \& Chen, J. The HARP-like domain-containing protein AH2/ZRANB3 binds to PCNA and participates in cellular response to replication stress. Mol. Cell 47, 410-421 (2012).

26. Weston, R., Peeters, H. \& Ahel, D. ZRANB3 is a structure-specific ATPdependent endonuclease involved in replication stress response. Genes Dev. 26, 1558-1572 (2012).

27. Ying, S., Hamdy, F. C. \& Helleday, T. Mre11-dependent degradation of stalled DNA replication forks is prevented by BRCA2 and PARP1. Cancer Res. 72, 2814-2821 (2012).

28. Ding, X. et al. Synthetic viability by BRCA2 and PARP1/ARTD1 deficiencies. Nat. Commun. 7, 12425 (2016).

29. Feng, Z. et al. Rad52 inactivation is synthetically lethal with BRCA2 deficiency. Proc. Natl Acad. Sci. USA 108, 686-691 (2011).

30. Wray, J., Liu, J., Nickoloff, J. A. \& Shen, Z. Distinct RAD51 associations with RAD52 and BCCIP in response to DNA damage and replication stress. Cancer Res. 68, 2699-2707 (2008).

31. Sotiriou, S. K. et al. Mammalian RAD52 functions in break-induced replication repair of collapsed DNA replication forks. Mol. Cell 64, 1127-1134 (2016).

32. Bhowmick, R., Minocherhomji, S. \& Hickson, I. D. RAD52 facilitates mitotic DNA synthesis following replication stress. Mol. Cell 64, 1117-1126 (2016).

33. Sullivan, K. et al. Identification of a small molecule inhibitor of RAD52 by structure-based selection. PLOS ONE 11, e0147230 (2016).

34. Kumar, J. K. \& Gupta, R. C. Strand exchange activity of human recombination protein Rad52. Proc. Natl Acad. Sci. USA 101, $9562-9567$ (2004).

35. Bi, B., Rybalchenko, N., Golub, E. I. \& Radding, C. M. Human and yeast Rad52 proteins promote DNA strand exchange. Proc. Natl Acad. Sci. USA 101, 9568-9572 (2004).

36. Rothenberg, E., Grimme, J. M., Spies, M. \& Ha, T. Human Rad52-mediated homology search and annealing occurs by continuous interactions between 
overlapping nucleoprotein complexes. Proc. Natl Acad. Sci. USA 105, 20274-20279 (2008)

37. Mazina, O. M., Keskin, H., Hanamshet, K., Storici, F. \& Mazin, A. V. Rad52 inverse strand exchange drives RNA-templated DNA double-strand break repair. Mol. Cell doi:https://doi.org/10.1016/j.molcel.2017.05.019 (2017).

38. Jensen, R. B., Carreira, A. \& Kowalczykowski, S. C. Purified human BRCA2 stimulates RAD51-mediated recombination. Nature 467, 678-683 (2010).

39. Tarsounas, M., Davies, D. \& West, S. C. BRCA2-dependent and independent formation of RAD51 nuclear foci. Oncogene 22, 1115-1123 (2003).

40. Ma, C. J., Gibb, B., Kwon, Y., Sung, P. \& Greene, E. C. Protein dynamics of human RPA and RAD51 on ssDNA during assembly and disassembly of the RAD51 filament. Nucleic Acids Res. 45, 749-761 (2017).

41. Bailis, J. M., Luche, D. D., Hunter, T. \& Forsburg, S. L. Minichromosome maintenance proteins interact with checkpoint and recombination proteins to promote s-phase genome stability. Mol. Cell Biol. 28, 1724-1738 (2008).

42. Hashimoto, Y., Puddu, F. \& Costanzo, V. RAD51- and MRE11-dependent reassembly of uncoupled CMG helicase complex at collapsed replication forks. Nat. Struct. Mol. Biol. 19, 17-24 (2011).

43. Huang, F. et al. Targeting BRCA1- and BRCA2-deficient cells with RAD52 small molecule inhibitors. Nucleic Acids Res. 44, 4189-4199 (2016).

44. Hengel, S. R. et al. Small-molecule inhibitors identify the RAD52-ssDNA interaction as critical for recovery from replication stress and for survival of BRCA2 deficient cells. Elife 5, e14740 (2016).

45. Sirbu, B. M., Couch, F. B. \& Cortez, D. Monitoring the spatiotemporal dynamics of proteins at replication forks and in assembled chromatin using isolation of proteins on nascent DNA. Nat. Protoc. 7, 594-605 (2012).

\section{Acknowledgements}

We thank the Center for Microscopy and Image Analysis of the University of Zurich for technical assistance with EM. We are grateful to B. Conti, A. Smogorzewska, and M. Jasin for sharing useful reagents and to all members of the Lopes group for useful discussions. This work was supported by the SNF grant 31003A 169959, the ERC Consolidator Grant 617102, and the Swiss Cancer League grant KFS-3967-08-2016 to M.L. and by the SNF grant 31003A_166451 to P.J. A.R.C. was supported by Human Frontier Science Fellowship (LT000393/2013), by the Center of Cancer Research and US Department of Defense (BCRP DOD Idea Expansion Award BC133858 and BCRP Break -through Award BC151331) to A.N.

\section{Author contributions}

S.M. performed and analyzed all EM, DNA fibers, iPOND, western blot, and fluorescence-activated cell sorting (FACS) experiments in RPE-1, U2OS and V-C8 Chinese hamster cells, with extensive technical assistance of R.Z., S.U., and K.M. N.C. performed all metaphase spread analyses, supervised by P.J. M.B. assisted with iPOND and with EM experiments on BJ and RAD51-T131P fibroblasts. K.J. helped performing key experiments with mouse ESCs. A.R.C. and A.N. provided crucial reagents ahead of publication and assisted with experimental design and manuscript finalization. M.L. designed and supervised the project and wrote the manuscript

\section{Additional information}

Supplementary Information accompanies this paper at doi:10.1038/s41467-017-01164-5.

Competing interests: The authors declare no competing financial interests.

Reprints and permission information is available online at http://npg.nature.com/ reprintsandpermissions/

Publisher's note: Springer Nature remains neutral with regard to jurisdictional claims in published maps and institutional affiliations.

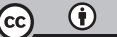

Open Access This article is licensed under a Creative Commons Attribution 4.0 International License, which permits use, sharing, adaptation, distribution and reproduction in any medium or format, as long as you give appropriate credit to the original author(s) and the source, provide a link to the Creative Commons license, and indicate if changes were made. The images or other third party material in this article are included in the article's Creative Commons license, unless indicated otherwise in a credit line to the material. If material is not included in the article's Creative Commons license and your intended use is not permitted by statutory regulation or exceeds the permitted use, you will need to obtain permission directly from the copyright holder. To view a copy of this license, visit http://creativecommons.org/ licenses/by/4.0/.

(c) The Author(s) 2017 